\title{
Survival of patients with newly diagnosed high-grade myeloid neoplasms who do not meet standard trial eligibility
}

\author{
Mary-Elizabeth M. Percival, ${ }^{1,2}$ Megan Othus, ${ }^{3}$ Sarah Mirahsani, ${ }^{1}$ \\ Kelda M. Gardner, ${ }^{1}$ Carole Shaw, ${ }^{2}$ Anna B. Halpern, ${ }^{1,2}$ Pamela S. Becker, ${ }^{1,2}$ \\ Paul C. Hendrie, ${ }^{1}$ Mohamed L. Sorror, ${ }^{2,4}$ Roland B. Walter ${ }^{1,2}$ \\ and Elihu H. Estey ${ }^{1,2}$
}

Haematologica 2021

Volume 106(8):2114-2120

${ }^{1}$ Division of Hematology, Department of Medicine, University of Washington; ${ }^{2}$ Clinical Research Division, Fred Hutchinson Cancer Research Center; ${ }^{3}$ Public Health Sciences, Fred Hutchinson Cancer Research Center and ${ }^{4}$ Division of Medical Oncology, Department of Medicine, University of Washington, Seattle, WA, USA

\section{ABSTRACT}

F lew patients with cancer, including those with acute myeloid leukemia and high-grade myeloid neoplasms, participate in clinical trials. Broadening standard eligibility criteria may increase clinical trial participation. In this retrospective single-center analysis, we identified 442 consecutive newly diagnosed patients from 2014 to 2016. Patients were considered "eligible" if they had a performance status $0-2$, normal renal and hepatic function, no recent solid tumor, left ventricular ejection fraction $(\mathrm{EF}) \geq 50 \%$, and no history of congestive heart failure (CHF) or myocardial infarction (MI); "ineligible" patients failed to meet one or more of these criteria. We included 372 patients who received chemotherapy. Ineligible patients represented $40 \%$ of the population and had a 1.79-fold greater risk of death (95\% Confidence Interval [CI]: 1.37-2.33) than eligible patients. Very few patients had cardiac comorbidities, including $2 \%$ with low EF, $4 \%$ with prior $\mathrm{CHF}$, and $5 \%$ with prior MI. In multivariable analysis, ineligibility was associated with decreased survival (Hazard ratio [HR] 1.44; 95\% CI: 1.07-1.93). Allogeneic transplantation, performed in 150 patients (40\%), was associated with improved survival (HR 0.66, 95\% CI: 0.48-0.91). Therefore,

\section{Correspondence:}

MARY-ELIZABETH M. PERCIVAL mperciva@uw.edu

Received: April 6, 2020.

Accepted: July 6, 2020.

Pre-published: July 9, 2020.

https://doi.org/10.3324/haematol.2020.254938

(C)2021 Ferrata Storti Foundation

Material published in Haematologica is covered by copyright. All rights are reserved to the Ferrata Storti Foundation. Use of published material is allowed under the following terms and conditions:

https://creativecommons.org/licenses/by-nc/4.0/legalcode. Copies of published material are allowed for personal or internal use. Sharing published material for non-commercial purposes is subject to the following conditions:

https://creativecommons.org/licenses/by-nc/4.0/legalcode, sect. 3. Reproducing and sharing published material for commercial purposes is not allowed without permission in writing from the publisher. standard eligibility characteristics identify a patient population with improved survival. Further treatment options are needed for patients considered ineligible for clinical trials.

\section{Introduction}

National Comprehensive Cancer Network guidelines state "the best management of any patient with cancer is in a clinical trial." Nonetheless, relatively few adults with cancer participate in clinical trials. ${ }^{1}$ Preferences of patients and physicians, and the distances separating patients from an academic center, undoubtedly contribute and are probably not amenable to change. In contrast, protocol eligibility criteria may be more flexible. These are often stereotypical, ignoring the complexity and individualized nature of clinical care. Oncology clinical trials, particularly those sponsored by industry, are generally written with standard eligibility (and ineligibility) criteria which if anything are becoming more stringent over time. ${ }^{2}$ The American Society of Clinical Oncology (ASCO) and similar groups have encouraged broader eligibility criteria, thus increasing the relevance of clinical trial results for the great majority of patients who do not participate in trials. ${ }^{3}$

An analysis by Lichtman et al., published simultaneously with the ASCO recommendations, found $50-85 \%$ of over 10,000 patients with breast, colorectal, lung, or bladder cancer seen in 2013 and 2014 at Kaiser Permanente Northern California would not meet standard clinical trial eligibility criteria. ${ }^{4}$ This analysis defined eligibility as meeting the requirements of younger age, relatively normal heart, kidney, and liver function, and no recent solid tumor. While some investigational 
agents may have only theoretical organ toxicity, the organ function parameters are infrequently adjusted or waived.

Clinical trial participation by patients with hematologic malignancies has received less attention. Statler et al. found patients retrospectively determined to be ineligible for Soutwest Oncology Group (SWOG) leukemia studies had similar outcomes as eligible patients treated on the same studies. However, many patients were found to be ineligible solely because of such administrative reasons as missing documentation or laboratory values drawn at the wrong time..$^{5}$ A more recent single-center analysis examining the effect of comorbidities and organ dysfunction found that a very high proportion of acute myeloid leukemia (AML) patients (88\%) would have been excluded from many clinical trials, but that outcomes did not differ significantly between eligible and ineligible patients. ${ }^{6}$ The authors therefore suggested that clinical trial eligibility criteria should be liberalized for AML patients.

In this single-center retrospective analysis, we examined the proportion of patients at our center with newlydiagnosed AML or high-grade myeloid neoplasms who would have met standard clinical trial eligibility criteria. We also compared outcome in patients according to whether they met these criteria.

\section{Methods}

\section{Patient population}

Consecutive patients diagnosed with AML or high-grade myeloid neoplasms (10-19\% blasts) at the University of Washington (UW)/Fred Hutchinson Cancer Research Center (FHCRC) between January 1, 2014 and December 31, 2016 were identified through our institutional database. The study was approved by the UW Institutional Review Board.

We identified 442 patients approximately equally divided between 2014, 2015, and 2016. Data on age, sex, performance status (PS), SWOG cytogenetic risk, ${ }^{7}$ treatment related mortality (TRM) score, ${ }^{8}$ induction treatment and intensity, and baseline values for glomerular filtration rate (GFR), creatinine, bilirubin, and alanine aminotransferase (ALT) were collected using the institional database. The TRM score uses pre-treatment patient and disease characteristics to estimate the probability of death within the first 28 days after induction. In a few patients, a single component for TRM calculation was missing so median values from the database as a whole were used. Patients were classified as having secondary disease if they had an antecedent hematologic disorder or previous exposure to chemotherapy. Induction treatment intensity was divided into three categories: high (containing cytarabine at $1 \mathrm{~g} / \mathrm{m}^{2} /$ dose or more); intermediate (including $7+3$, CPX-351, or similar); and low (hypomethylating agents, low-dose cytarabine, or similar). We excluded patients from subsequent analysis who received either palliative care alone or unknown treatment in the community $(n=70 ; 16 \%$ of the entire population). Additional information regarding prior malignancy (excluding prior AML, myelodysplastic syndrome, myeloproliferative neoplasms, and non-melanoma skin cancer), cardiac disease and/or low ejection fraction (EF) within 3 months of planned treatment, were collected from review of medical charts (performed by MEP). Response to induction chemotherapy was defined as complete remission (CR; absolute neutrophil count [ANC] $>1,000 / \mu \mathrm{L}$ and platelet count $<100,000 / \mu \mathrm{L}$ ), CR with incomplete count recovery (CRi; ANC
$<1,000 / \mu \mathrm{L}$ ), or CR with incomplete platelet recovery (CRp; platelet count $<100,000 / \mu \mathrm{L}$ ). Measurable residual disease (MRD) was assessed using multiparameter flow cytometry.

\section{Definition of eligibility for clinical trials}

Patients were considered "eligible" for the purposes of our retrospective analysis had PS $0-2$, GFR $\geq 60 \mathrm{ml} / \mathrm{min}$, ALT $\leq$ twice the upper limit of normal (ULN), bilirubin $\leq 1.5 \mathrm{mg} / \mathrm{dL}$, no solid tumor diagnosed within 2 years preceding the diagnosis of $\mathrm{AML}$, left ventricular ejection fraction $(\mathrm{EF}) \geq 50 \%$, and no history of congestive heart failure (CHF) or myocardial infarction (MI). The criteria are similar to those in a previous analysis of solid tumor patients and mimic standard trial eligibility criteria. ${ }^{4}$ Patients were classified as "ineligible" if they failed to meet at least one of these eligibility criteria. Unknown values were not considered to make a patient ineligible. Though some trials exclude patients with older age, we did not use age as a criterion in light of the recent drug approvals in AML., 10

\section{Statistical analysis}

Overall survival (OS) was measured from date of first evaluation to date of death, with patients last known to be alive censored at the date of last contact. Fisher's exact tests and Wilcoxon rank-sum tests were used to compare eligible and ineligible patients. Fisher's exact test was used to evaluate 28-day mortality; patients censored before day 28 were excluded from the analysis. Fisher's exact test was used to evaluate response; response was not available on 29 patients. Cox regression models and log-rank tests were used to evaluate associations with OS. All statistical analyses were performed using R. ${ }^{11}$

\section{Results}

\section{Baseline characteristics}

We identified 372 patients; 272 patients received intermediate or high intensity induction and 100 low intensity induction (Table 1). Of these, 207 patients (56\%) received treatment on a clinical trial at UW/FHCRC.

Using the above-noted criteria (performance status, GFR, ALT, bilirubin, prior solid tumor within 2 years, EF, and history of CHF or MI), 220 patients (60\%) would have been considered eligible. The reasons for ineligibility are shown in Table 1 . Typically, for a given eligibility criterion $<10 \%$ of patients were ineligible. For example, only $2 \%$ of patients would have been ineligible based on the usual requirement for $\mathrm{EF} \geq 50 \%$, only $4 \%$ because of prior CHF, only $5 \%$ because of prior MI, only $5 \%$ because of elevated ALT, and only $5 \%$ because of abnormal bilirubin. Only $3 \%$ of patients would have been ineligible because they had a solid tumor in the last 2 years, which is probably the most common solid-tumor free interval included in clinical trial eligibility criteria.

\section{Relationship between ineligibility and survival}

Univariate analyses showed strong associations between risk of death and ineligibility criteria including PS 3-4, GFR $<60 \mathrm{~mL} / \mathrm{min}$, and prior CHF or MI (Table 1). Associations between survival and abnormal ALT, abnormal bilirubin, or decreased EF were not noted, but there were relatively few patients in these categories. Patients with one or more ineligibility characteristics represented approximately $40 \%$ of the study population and had a 1.79 -fold greater risk of death $(95 \%$ Confidence Interval 
Table 1. Univariate associations between baseline characteristics and overall survival. Median overall survival (OS) (years) based on Kaplan-Meier estimates, and hazard ratios (HR), 95\% Confidence Intervals (Cl), and $P$-values from univariate Cox regression models reported. Median 0S for treatment related mortality (TRM) is reported for all patients.

\begin{tabular}{|c|c|c|c|c|}
\hline Factor & Summary & Medlan OS (95\% Cl) (years) & HR (95\% CI) & $P$ \\
\hline 2014 & $114(31)$ & $1.5(1.1,3.0)$ & Reference & - \\
\hline 2015 & $133(36)$ & $1.2(1.0,2.0)$ & $1.23(0.9,1.68)$ & 0.2 \\
\hline 2016 & $125(34)$ & $1.8(1.1,2.5)$ & $1.05(0.75,1.47)$ & 0.78 \\
\hline Female & $154(42)$ & $2.2(1.5,2.8)$ & Reference & - \\
\hline Male & $216(58)$ & $1.2(1.0,1.5)$ & $1.31(1,1.71)$ & 0.049 \\
\hline AML & $264(71 \%)$ & $1.5(1.1,2.2)$ & Reference & \\
\hline High grade myeloid neoplasm (<20\% blasts) & $107(29 \%)$ & $1.4(1.1,2.1)$ & $0.94(0.71,1.24)$ & 0.64 \\
\hline PS 0-1 & $300(81)$ & $1.8(1.4,2.4)$ & Reference & - \\
\hline PS 2 & $53(14)$ & $0.7(0.6,1.4)$ & $1.77(1.25,2.5)$ & 0.0011 \\
\hline PS 3-4 & $19(5)$ & $0.2(0.1$, inf $)$ & $2.73(1.61,4.63)$ & $<0.001$ \\
\hline De novo & $181(49)$ & $2.0(1.3,3.0)$ & Reference & - \\
\hline Secondary & $191(51)$ & $1.2(1.0,1.8)$ & $1.41(1.08,1.83)$ & 0.01 \\
\hline Favorable cytogenetic risk & $19(5)$ & NR $(1.6$, inf $)$ & Reference & - \\
\hline Intermediate cytogenetic risk & $226(61)$ & $1.8(1.3,2.4)$ & $2.17(0.96,4.93)$ & 0.063 \\
\hline Adverse cytogenetic risk & $114(31)$ & $0.9(0.6,1.4)$ & $3.4(1.48,7.8)$ & 0.0038 \\
\hline Unknown cytogenetic risk & $13(3)$ & $0.4(0.1$, inf $)$ & $2.62(0.88,7.8)$ & 0.083 \\
\hline TRM score (per 1 point increase in score) & $4.59(0,73.25)$ & $1.4(1.2,2.0)$ & $1.05(1.04,1.05)$ & $<0.001$ \\
\hline On study & $207(56)$ & $1.4(1.1,2.3)$ & Reference & - \\
\hline Not on study & $165(44)$ & $1.4(1.0,2.0)$ & $1.1(0.85,1.43)$ & 0.47 \\
\hline High intensity therapy & $212(57)$ & $2.4(1.8,3.5)$ & Reference & - \\
\hline Intermediate intensity therapy & $60(16)$ & $0.7(0.4,1.6)$ & $2.29(1.61,3.26)$ & $<0.001$ \\
\hline Low intensity therapy & $100(27)$ & $1.1(0.9,1.4)$ & $1.93(1.44,2.59)$ & $<0.001$ \\
\hline Age $\leq 75$ & $319(86)$ & $1.9(1.4,2.4)$ & Reference & - \\
\hline Age $>75$ & $53(14)$ & $0.7(0.5,1.0)$ & $2.38(1.72,3.3)$ & $<0.001$ \\
\hline ALT $\leq 2 x$ ULN & $349(95)$ & $1.4(1.2,2.0)$ & Reference & - \\
\hline ALT $>2 x$ ULN & $17(5)$ & $0.7(0.6$, inf $)$ & $1.11(0.59,2.09)$ & 0.75 \\
\hline $\mathrm{GFR} \geq 60 \mathrm{ml} / \mathrm{min}$ & $319(87)$ & $1.8(1.3,2.3)$ & Reference & - \\
\hline $\mathrm{GFR}<60 \mathrm{ml} / \mathrm{min}$ & $46(13)$ & $0.8(0.5,1.8)$ & $1.73(1.2,2.49)$ & 0.0035 \\
\hline Bilirubin $\leq 1.5 \mathrm{mg} / \mathrm{dL}$ & $347(95)$ & $1.5(1.2,2.1)$ & Reference & - \\
\hline Bilirubin $>1.5 \mathrm{mg} / \mathrm{dL}$ & $20(5)$ & 0.7 (0.1, inf) & $1.49(0.87,2.56)$ & 0.15 \\
\hline No solid tumor in prior 2 years & $353(97)$ & $1.4(1.2,2.1)$ & Reference & - \\
\hline Solid tumor within prior 2 years & $10(3)$ & $2.0(1.3$, inf $)$ & $0.84(0.35,2.05)$ & 0.7 \\
\hline $\mathrm{EF} \geq 50 \%$ & $275(98)$ & $1.8(1.2,2.4)$ & Reference & - \\
\hline $\mathrm{EF}<50 \%$ & $5(2)$ & 3.5 (0.0, inf) & $1(0.32,3.15)$ & 1 \\
\hline No prior CHF & $359(96)$ & $1.5(1.2,2.1)$ & Reference & - \\
\hline Prior CHF & $14(4)$ & 0.6 (0.2, inf) & $1.9(1.04,3.49)$ & 0.037 \\
\hline No prior MI & $353(95)$ & $1.6(1.2,2.2)$ & Reference & - \\
\hline Prior MI & $19(5)$ & $1.0(0.5,0.6)$ & $1.93(1.18,3.18)$ & 0.0091 \\
\hline
\end{tabular}

PS: performance status; AML: acute myeloid leukemia; OS: overall survival; TRM: treatment-related mortality; ALT: alanine aminotransferase; ULN: upper limit of normal; GFR glomerular filtration rate; EF: ejection fraction; CHF: congestive heart failure; MI: myocardial infarction; HR: hazard ratio; CI: Confidence Interval; inf: infinity; NR: not reached.

[CI]: 1.37-2.33) than patients who had no ineligibility characteristics (Table 2; Figure 1). Of the 144 ineligible patients, $73 \%$ (106) failed to meet only a single eligibility criterion, $26 \%$ did not meet two eligibility criteria, while $5 \%$ did not meet three criteria (Table 2 ).

\section{Relationship between ineligibility and baseline characteristics (Table 3)}

Although we did not regard secondary AML as a criterion of ineligibility per se, patients with secondary AML were more likely to be ineligible $(P<0.001)$. This is likely because many of the secondary AML patients were classified as such due to receipt of chemotherapy for a prior malignancy, which in itself is a common reason for exclusion from trials. As expected, there were strong associations between ineligibility and higher TRM scores $(P<0.001)$. Nonetheless, rate of 28 -day mortality was similar between eligible and ineligible patients (4\% vs. $8 \%$, $P=0.06$ ). Also as expected, ineligible patients were more likely to receive intermediate and less intense induction therapy, rather than more intense induction $(P=0.006)$. Although we observed no association between eligibility 
Table 2. Univariate associations between ineligibility and overall survival.

\begin{tabular}{lccc} 
Factor & N $(\%)$ & HR $(95 \%$ CI) & $P$ \\
No ineligible characteristic & $220(60)$ & Reference & - \\
1 ineligible characteristic & $106(29)$ & $1.68(1.26,2.25)$ & $<0.001$ \\
2 ineligible characterisics & $31(9)$ & $2.12(1.34,3.34)$ & 0.0013 \\
3 ineligible characteristics & $7(2)$ & $2.26(1,5.14)$ & 0.051 \\
No ineligible characteristic & $220(60)$ & Reference & - \\
1 or more ineligible characteristic & $144(40)$ & $1.79(1.37,2.33)$ & $<0.001$ \\
\hline HR: Hazard ratio; CI: Confidence Interval. & &
\end{tabular}

and cytogenetic risk, the rate of CR without MRD was significantly higher in eligible patients (55\% vs. 38\%). Partly as a consequence, although eligible patients comprised $60 \%$ of our population, they comprised $74 \%$ of the 140 patients who received allogeneic hematopoietic cell transplantation (HCT) $(P=0.004)$. It is interesting to observe that 39 "ineligible" patients went on to allogeneic HCT, but notably 30 met only one ineligibility criterion at diagnosis.

\section{Ineligibility is associated with decreased survival (Table 4)}

A multivariable Cox regression model indicated that the presence of one more ineligible factors was associated with decreased overall survival (HR 1.45, 95\% CI: 1.081.93) (Table 4), even after accounting for known prognostic factors. Considered as a time-dependent variable, HCT was associated with improved survival (HR 0.67, 95\% CI: 0.49-0.93). Tests of interaction between HCT and ineligibility indicated no evidence of a difference in the association between transplant and outcome between eligible and ineligible patients (HR 0.99, $P=0.99$ ).

We also examined the effect of participation in clinical trials in our cohort. Many of the clinical trials on which our patients were enrolled were investigator-initiated trials which commonly had more lenient inclusion criteria than the "standard" eligibility criteria defined for the current analysis, perhaps leading our trial patients to have worse prognoses than those more typically enrolled. Examples of clinical trials enrolling during this time period that targeted patients with a high treatment-related mortality include reduced-dose CPX-3512 and a randomized trial for reduced versus full-dose CLAG-M. ${ }^{13}$ In fact, despite the relation between shorter survival and ineligibility for clinical trials, participation in a clinical trial (207 patients, $56 \%$ of the cohort) was not associated with improvement in survival (HR 1.01, 95\% CI: 0.76-1.33). Furthermore, of the 207 patients treated on study, 74 $(36 \%)$ were ineligible by at least one criterion, and the effect of eligibility on overall survival was similar in patients treated on or off a trial (interaction $P=0.15$ ).

\section{Discussion}

Because overly strict eligibility criteria for clinical trials may lead to unrepresentative study populations, we set out to examine the frequency of characteristics associated with ineligibility. We observed a high percentage of patients with at least one ineligible characteristic (40\%), though this finding is somewhat less than the $50-85 \%$
Table 3. Univariate associations between baseline characteristics and ineligibility. Median (range) or numbers (N) (\%) reported for summary.

\begin{tabular}{|c|c|c|c|}
\hline Factor & $\begin{array}{c}\text { No ineligible } \\
\text { characteristic } \\
(n=220)\end{array}$ & $\begin{array}{c}1 \text { or more } \\
\text { inelighlole } \\
\text { characteristics } \\
\text { (n=144) }\end{array}$ & $P$ \\
\hline 2014 & $68(31)$ & $42(29)$ & 0.77 \\
\hline 2015 & $76(35)$ & $55(38)$ & \\
\hline 2016 & $76(35)$ & $47(33)$ & \\
\hline Female & $98(45)$ & $52(36)$ & 0.13 \\
\hline Male & $121(55)$ & $91(64)$ & \\
\hline De novo & $135(61)$ & $45(31)$ & $<0.001$ \\
\hline Secondary & $85(39)$ & $99(69)$ & \\
\hline Favorable cytogenetic risk & $12(5)$ & $7(5)$ & 0.96 \\
\hline Intermediate cytogenetic risk & $134(61)$ & $86(60)$ & \\
\hline Adverse cytogenetic risk & $67(30)$ & $45(31)$ & \\
\hline Unknown cytogenetic risk & $7(3)$ & $6(4)$ & \\
\hline TRM score & $4(0,73)$ & $6(0,73)$ & $<0.001$ \\
\hline High intensity therapy & $143(65)$ & $69(48)$ & 0.0035 \\
\hline Intermediate intensity therapy & $31(14)$ & $28(19)$ & \\
\hline Low intensity therapy & $46(21)$ & $47(33)$ & \\
\hline Alive past day 28 & $209(96)$ & $130(92)$ & 0.06 \\
\hline Died on or before day 28 & $8(4)$ & $12(8)$ & \\
\hline CR without MRD & $121(55)$ & $54(38)$ & 0.014 \\
\hline CR with MRD & $24(11)$ & $17(12)$ & \\
\hline CRi/CRp with or without MRD & $28(13)$ & $23(16)$ & \\
\hline Refractory & $34(15)$ & $35(24)$ & \\
\hline Missing & $13(6)$ & $15(10)$ & \\
\hline
\end{tabular}

TRM: treatment-related mortality; CR: complete remission; CRi: $\mathrm{CR}$ with incomplete neutrophil recovery; CRp: CR with incomplete platelet recovery; MRD: measurable residual disease, as detected by multiparameter flow cytometry.

ineligible patients identified in the Lichtman analysis of solid tumor trial candidates and less than the $88 \%$ ineligible identified in the Statler analysis of AML patients treated with chemotherapy.,6 Our analysis excluded a subset of patients who received palliative care alone or unknown treatment in the community. Because of the nature of oncology trials, the Lichtman analysis notably also included age over 75 as an exclusion criterion, which was a characteristic of $14 \%$ of our final cohort.

Cardiac disease was uncommon in our cohort, at least based on history of CHF (4\%) or MI $(5 \%)$ and decreased EF within 3 months of leukemia diagnosis (2\%). The Lichtman analysis was comparable, with CHF/cardiomyopathy observed in $5-11 \%$ of patients and prior MI in 1$5 \%$, depending on cancer type. ${ }^{4}$ In our analysis, EF assessment was done on a routine basis for most patients prior to planned receipt of an anthracycline during induction chemotherapy, even though most cardiac toxicity is only seen after high cumulative anthracycline doses. ${ }^{14} \mathrm{EF}$ evaluation can be expensive and can delay the initiation of life-saving chemotherapy, and these results suggest that it is unnecessary more often than not. ${ }^{15}$ In contrast, a previous analysis of 97 randomized controlled trials in hematologic malignancies, not limited to AML and MDS, determined that cardiac eligibility criteria were significantly associated with observed adverse events in a way that hepatic and renal eligibility criteria were not. ${ }^{16}$ 


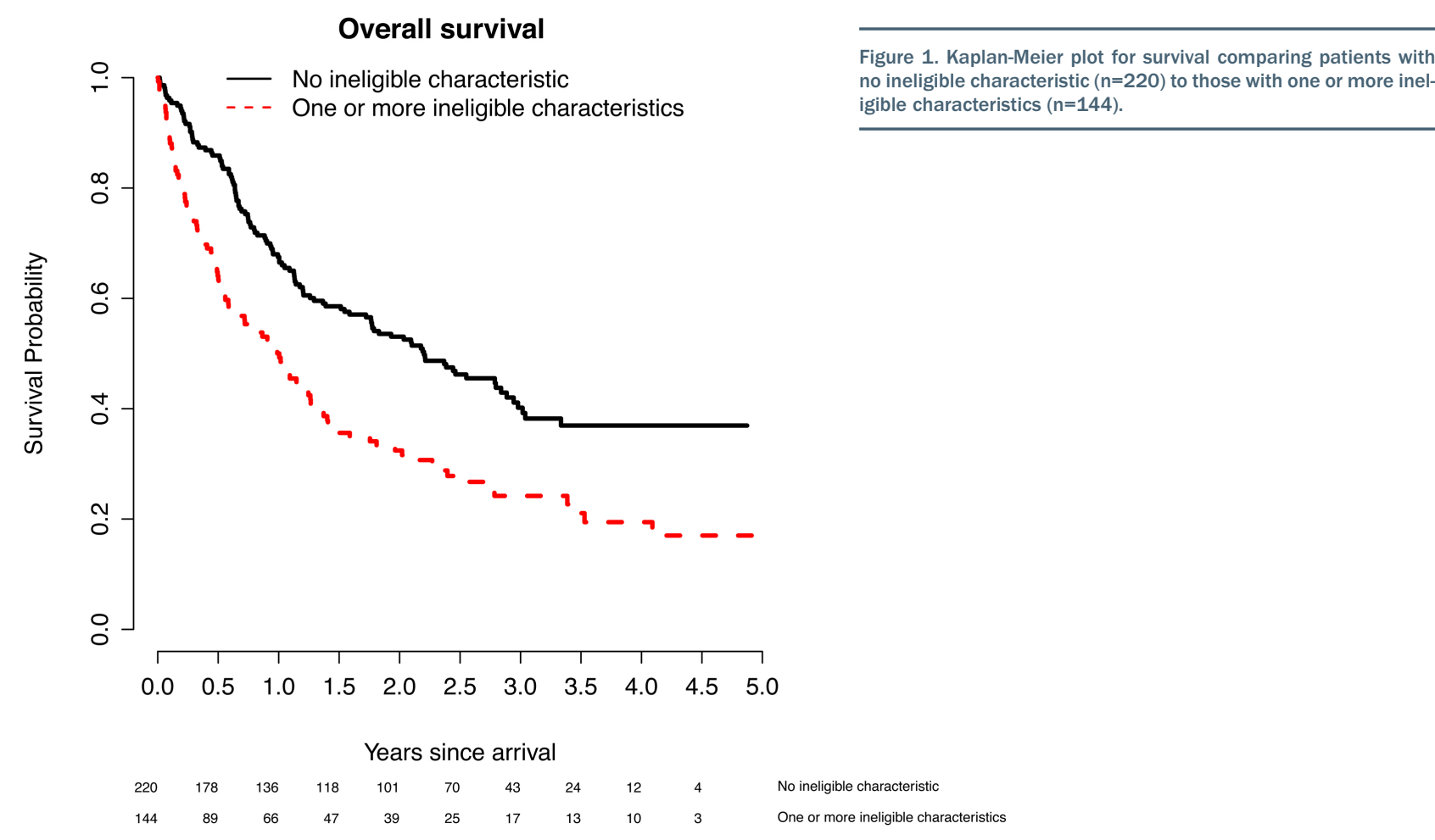

Table 4. Multivariable Cox regression model with time-dependent transplant variable.

\begin{tabular}{lccc}
\hline Covariate & IR & $95 \%$ CI & \\
Transplant (ref = no transplant) & 0.67 & $(0.49,0.93)$ & 0.016 \\
Male (ref = female) & 1.15 & $(0.87,1.15)$ & 0.33 \\
\hline Secondary AML (ref = de novo) & 1 & $(0.75,1.32)$ & 1 \\
Intermediate cytogenetic risk (ref = favorable) & 1.97 & $(0.86,4.52)$ & 0.11 \\
\hline Adverse risk (ref = favorable) & 3.29 & $(1.4,7.73)$ & 0.0063 \\
Unknown risk (ref = favorable) & 2.97 & $(0.99,8.95)$ & 0.052 \\
\hline Age (years) & 1.02 & $(1.01,1.04)$ & $<0.001$ \\
Intermediate intensity therapy (ref = high intensity) & 2.08 & $(0.77,1.54)$ & $<0.001$ \\
\hline Low intensity (ref = high intensity) & 1.09 & $(0.86,1.49)$ & 0.63 \\
Not on study (ref = on study) & 1.13 & $(1.08,1.93)$ & 0.37 \\
\hline One or more ineligible factors (ref = no ineligible factors) & 1.45 & 0.012 \\
\hline AML
\end{tabular}

AML: acute myeloid leukemia;TRM: treatment-related mortality; HR: hazard ratio; CI: Confidence Interval; ref: reference.

Cardiac eligibility criteria may act as a surrogate for other co-morbidities and may predict toxicity, but the authors of that study conclude that exclusion criteria are often too broad and not applicable.

In our analysis, patients with at least one ineligible characteristic had significantly worse survival than patients with no ineligible characteristic (HR 1.79, 95\% CI: 1.37-2.33; Table 4). It is likely that all the criteria used to determine ineligibility are not equally unfavorable. Optimally, our models would analyze each criterion separately, rather than combining them together as in this study. However, estimating the contribution of individual ineligibility characteristics would require a larger patient cohort. Our finding that ineligibility is associated with decreased survival suggests that standard clinical trial criteria may identify a population of patients with better outcomes following treatment; improved responses and survival have also been demonstrated in an "on study" population when the same induction regimen was administered to patients both on and off clinical trial at our center and in a national population-based cohort study from Denmark. ${ }^{17,18}$ These findings contrast some with the Statler analysis of AML patients, which did not identify significant differences in response or survival based on comorbidities other than liver disease and organ dysfunction. ${ }^{6}$ In the future, evaluation of other factors including travel distance, social support, and frailty level could provide a more nuanced picture of eligibility.

Before performing our analysis, we believed ineligibility would be associated with worse outcomes largely because of its association with early death, or 28-day TRM, based on the sharp decline in death rate after this 28-day period. ${ }^{8}$ However, though ineligible patients had higher TRM scores and would be more likely to incur 
$\mathrm{TRM}^{8}$ the difference in TRM between eligible and ineligible patients was small when contrasted with the much greater differences in survival. This suggests much of the survival difference reflected the considerably higher rate of CR without MRD in eligible patients ( $55 \%$ vs. $38 \%$ ), since achievement of CR without MRD is associated with longer survival. ${ }^{19}$ Since cytogenetic risk was similar in eligible and ineligible patients and since none of our ineligibility criteria are known to be associated with resistance to therapy, another explanation is needed for this higher CR without MRD rate. One possibility is the much less frequent use of intense induction in ineligible patients. Ineligible patients were also much less likely to receive allogeneic HCT. Hence while electing not to give ineligible patients intense induction may reduce TRM, this effect may be considerably less than the entailed loss of efficacy (e.g., decreased rate of CR without MRD) and resultant loss of ability to receive HCT.

Most patients with AML are given standard treatment (off trial) in community centers. An important question is whether the effect of "ineligibility" is the same in community and academic centers. Patients treated in community centers are older, have more comorbidities, and travel less distance for treatment than those at academic centers. ${ }^{20}$ Although after accounting for these factors survival remained better in academic centers, the negative effect of covariates such as comorbidities and shorter distance traveled was similar in both settings, suggesting that the effect of ineligibility is similar in community and academic centers. Nonetheless, the pre-selection of patients inherent in a retrospective study from an academic medical center undoubtedly adds to the effect of ineligibility in evaluating the relevance of clinical trial outcomes.

As noted in the results, many of the patients entered on trials at our center would have been ineligible for typical trials. The lack of a linkage between participation in a trial and fulfillment of standard eligibility criteria may partially explain the failure of our trials to improve survival, although the trials themselves may have been at fault. Though participation in clinical trials is encouraged, it is also true that not all trials lead to improved outcomes for patients. Patients with characteristics that make them ineligible for most trials may be the very population who would benefit most from novel therapies that are available only within the context of a trial. Additionally, improvements in supportive care over time will benefit all patients, regardless of eligibility. Investigators at $\mathrm{MD}$ Anderson Cancer Center published the results of a clinical trial for patients with AML and MDS who were by definition not eligible for standard clinical trials, ${ }^{21}$ but we are not aware of any other similar studies either completed or enrolling. Future studies with broader inclusion criteria could also include a "correction factor" to help compensate for the expected worse outcomes of patients who would not meet standard eligibility. The recent Food and Drug Admistration approvals in the AML arena have changed the landscape of treatment options, but the real world use of these drugs may not mimic the cohort studied in the clinical trials leading to approval.

Even the possibility of less benefit for "ineligible" patients may not be sufficient justification for their continued exclusion from trials. For many patients with AML, standard treatment is unsatisfactory. Hence, once properly informed, many patients would prefer to enroll on trials rather than receive standard therapy. Extended to many cancers, this fact underlies the "right-to-try" movement. We understand patients are often ineligible for trials because the trials' sponsors realize inclusion would lead to less encouraging results, harming chances for regulatory approval. A possible means to reconcile the interests of patients and of sponsors would be to make regulatory approval of new drugs might be made conditional on subsequent conduct of trials in patients underrepresented in the trials prompting initial approval, thus potentially increasing the applicability of results to the substantial numbers of patients currently considered "ineligible."

\section{Disclosures}

This study was presented, in part, at the American Society of Hematology 2019 annual meeting in Orlando, FL, USA.

\section{Contributions}

MEMP and EHE: conceived and designed the study; data collection was performed by MEMP, SM, KMG, and CS; statistical analysis was performed by $M O$. The remaining authors enrolled patients and provided feedback and revisions. The final manuscript was approved by all authors.

\section{Funding}

The study was supported in part by a Cancer Consortium Grant from the National Institutes of Health (P30 CA015704).

\section{References}

1. Network NCC. Acute Myeloid Leukemia (version 2.2019). https://www.nccn.org/ professionals/physician_gls/pdf/aml.pdf. Accessed April 12, 2019

2. Srikanthan A, Vera-Badillo F, Ethier J, et al. Evolution in the eligibility criteria of randomized controlled trials for systemic cancer therapies. Cancer Treat Rev. 2016; 43:67-73.

3. Kim ES, Bruinooge SS, Roberts S, et al. Broadening eligibility criteria to make clinical trials more representative: American Society of Clinical Oncology and Friends of Cancer Research Joint Research Statement. J Clin Oncol. 2017;35(33):3737-3744.

4. Lichtman SM, Harvey RD, Damiette Smit $\mathrm{MA}$, et al. Modernizing clinical trial eligibility criteria: recommendations of the American Society of Clinical OncologyFriends of Cancer Research Organ
Dysfunction, Prior or Concurrent Malignancy, and Comorbidities Working Group. J Clin Oncol. 2017;35(33):37533759.

5. Statler A, Othus M, Erba HP, et al. Comparable outcomes of patients eligible vs ineligible for SWOG leukemia studies. Blood. 2018;131(25):2782-2788.

6. Statler A, Hobbs BP, Radivoyevitch T, et al. The impact of comorbidities and organ dysfunction commonly used for clinical trial eligibility criteria on outcome in acute myeloid leukemia (AML) patients receiving induction chemotherapy. Blood. 2019; 134(Suppl 1):S16.

7. Slovak ML, Kopecky KJ, Cassileth PA, et al. Karyotypic analysis predicts outcome of preremission and postremission therapy in adult acute myeloid leukemia: a Southwest Oncology Group/Eastern Cooperative Oncology Group Study. Blood. 2000; 96(13):4075-4083.
8. Walter RB, Othus M, Borthakur G, et al Prediction of early death after induction therapy for newly diagnosed acute myeloid leukemia with pretreatment risk scores: a novel paradigm for treatment assignment. J Clin Oncol. 2011;29(33):4417-4423.

9. Cortes JE, Heidel FH, Hellmann A, et al. Randomized comparison of low dose cytarabine with or without glasdegib in patients with newly diagnosed acute myeloid leukemia or high-risk myelodysplastic syndrome. Leukemia. 2019; 33(2):379-389.

10. DiNardo CD, Pratz K, Pullarkat V, et al. Venetoclax combined with decitabine or azacitidine in treatment-naive, elderly patients with acute myeloid leukemia. Blood. 2019;133(1):7-17.

11. R: A language and environment for statistical computing. R Foundation for Statistical Computing [computer program]. R Foundation for Statistical Computing, 
Vienna, Austria; 2017

12. Walter RB, Othus M, Orlowski KF, et al. Unsatisfactory efficacy in randomized study of reduced-dose CPX-351 for medically less fit adults with newly diagnosed acute myeloid leukemia or other highgrade myeloid neoplasm. Haematologica. 2018;103(3):e106-e109.

13. Halpern AB, Othus M, Gardner KM, et al. Mini- Vs. Regular-dose CLAG-M (Cladribine, Cytarabine, G-CSF, and Mitoxantrone) in medically less fit adults with newly-diagnosed acute myeloid leukemia (AML) and other high-grade myeloid neoplasms. Blood. 2019;134(Suppl 1):S1364.

14. Anderlini P, Benjamin RS, Wong FC, et al. Idarubicin cardiotoxicity: a retrospective study in acute myeloid leukemia and myelodysplasia. J Clin Oncol. 1995;13(11): 2827-2834

15. Khan HM, Gardner KM, Shaw C, et al. Need for routine examination of left ventricular ejection fraction in patients with AML. Leukemia. 2020;34(4):1169-1171.

16. Statler A, Radivoyevitch T, Siebenaller C, et al. The relationship between eligibility criteria and adverse events in randomized controlled trials of hematologic malignancies. Leukemia. 2017;31(8):1808-1815.

17. Buckley SA, Percival ME, Othus M, et al A comparison of patients with acute myeloid leukemia and high-risk myelodysplastic syndrome treated on versus off study. Leuk Lymphoma. 2019;60 (4):1023-1029.

18. Ostgard LS, Norgaard M, Sengelov $H$, et al. Improved outcome in acute myeloid leukemia patients enrolled in clinical trials a national population-based cohort study of Danish intensive chemotherapy patients. Oncotarget. 2016;7(44):7204472056.

19. Chen X, Xie H, Wood BL, et al. Relation of clinical response and minimal residual disease and their prognostic impact on outcome in acute myeloid leukemia. J Clin Oncol. 2015;33(11):1258-1264.

20. Bhatt VR, Shostrom V, Giri S, et al. Early mortality and overall survival of acute myeloid leukemia based on facility type. Am J Hematol. 2017;92(8):764-771.

21. Montalban-Bravo G, Huang X, Naqvi K, et al. A clinical trial for patients with acute myeloid leukemia or myelodysplastic syndromes not eligible for standard clinical trials. Leukemia. 2017;31(2):318-324. 